\title{
Comorbidities in patients with gout in rheumatology clinic Dr. Hasan Sadikin general hospital in 2012 - 2013
}

\author{
WR Limanjaya' ${ }^{1}$, RG Wachjudi², H Tansah ${ }^{3}$
}

${ }^{1}$ Faculty of Medicine,

Padjadjaran University

${ }^{2}$ Department of

Internal Medicine,

Faculty of Medicine,

Dr. Hasan Sadikin

General Hospital

${ }^{3}$ Department of

Biochemistry and

Biomolecular,

Faculty of Medicine,

Padjadjaran University

\begin{abstract}
Background: Gout is a metabolic disease manifested mainly as an intense monoarticular inflammatory reaction which is strongly associated with

hyperuricemia. Latest evidence showed that uric acid exerted effects on the development of other diseases. Many studies in developed countries had estimated the frequency of comorbidities associated with gout such as hypertension, obesity, diabetes mellitus, Chronic Kidney Disease (CKD), and Myocardial Infarct (MCI). However, no data regarding these frequencies have been found in Indonesia up to now to the best of the author's knowledge. This study aimed to establish the frequency of these comorbidities in patients with gout in Rheumatology Clinic Dr. Hasan Sadikin General Hospital, Bandung, Indonesia.
\end{abstract}

Methods: All medical records of patients with gout in Rheumatology Clinic Dr. Hasan Sadikin General Hospital from January 2012 to December 2013 were collected. The data on blood pressure; Body Mass Index (BMI); random blood glucose, fasting blood glucose or 2 hours post prandial blood glucose; history of myocardial infarction; and creatinine were taken and analyzed to determine the presence of comorbidities

Results: Among all patients with gout in Rheumatology Clinic Dr. Hasan Sadikin General Hospital, 53.08\% had chronic kidney disease, $42.73 \%$ had hypertension, $25.39 \%$ had diabetes mellitus, $15.70 \%$ had myocardial infarction and $12.22 \%$ had obesity.

Conclusions: Comorbidities commonly found in patients with gout in order of frequency were chronic kidney disease, hypertension, diabetes mellitus, myocardial infarct, and obesity.

Keywords: gout, comorbidities, frequency.

\section{Introduction}

Gout is a chronic disease affecting multiple systems which is caused by increased serum uric acid level (hyperuricemia). This condition contributed by error in purine metabolism or excretion is commonly further worsened by excessive purine intake. ${ }^{1}$ Hyperuricemia leads to deposition and nucleation of uric acid into Monosodium Urate (MSU) crystals in synovial space which induces an inflammatory reaction and clinically manifested as an acute and very painful arthritis commonly known as gouty arthritis. Extremely or chronically elevated serum uric acid level also can lead to several derangements in the renal including chronic urate nephropathy, acute uric acid nephropathy, and uric acid nephrolithiasis which eventually result in renal failure. $^{2}$

Increasing concern into this disease was brought by the fact that the prevalence of gout and hyperuricemia remain large and had risen in the past 2 decades. ${ }^{3}$ During its natural course of disease, patients with gout and hyperuricemia are also associated with many comorbidities such as hypertension, Chronic Kidney Disease (CKD), obesity, diabetes, and Myocardial Infarct (MCI) probably due to recently found role of uric acid in pathogenesis of these comorbidities. ${ }^{4}$ Several studies had been performed in United States, United Kingdom, and Germany to estimate the frequency of comorbidities in gout population. ${ }^{5,6}$ However, to the best of the author's knowledge, similiar data in Asia, particularly in Indonesia hasn't been documented. To address this issue, this study was designed to identify the frequency of comorbidities in patients with gout in Rheumatology Clinic Dr. Hasan Sadikin General Hospital, Bandung, Indonesia.

\section{Methods}

In order to know the pattern of comorbidities in patients with gout, cross sectional study using all medical records of such patients in Rheumatology Clinic Dr. Hasan Sadikin General Hospital were carried out. The medical record of a patient would be included if the time of admission was between January 2012 - December 2013 and the patient was diagnosed with gout by attending physician or given code as ICD (International Classification of Disease) M10. Diagnostic criteria used for gout in Rheumatology Clinic Dr. Hasan Sadikin General Hospital were based on American College of Rheumatology guidelines. Several data were recorded for data analysis purpose such as age, gender, weight in kilograms, height in centimeters, blood pressure at the time of admission, blood glucose test (fasting blood glucose, random blood glucose, or 2 hours post prandial blood glucose), serum creatinine level, and history of myocardial infarction. All the data collected were then inserted into Microsoft Excel for analysis. Patients without any of the data above were excluded

The presence of particular comorbidites was checked according to the following definitions. Hypertension was defined as systolic blood pressure 
more than or equal to $140 \mathrm{mmHg}$ or diastolic pressure more than or equal to $90 \mathrm{mmHg}$. $^{7}$ Obesity was defined as Body Mass Index $(\mathrm{BMI}) \geq 30 \mathrm{~kg} / \mathrm{m}^{2}$ where BMI is body weight divided by height in meters squared. ${ }^{8}$ Diabetes was defined as either fasting blood glucose $\geq 126 \mathrm{mg} / \mathrm{dl}$ or the presence of diabetic symptoms (polydipsia, polyuria, polyphagia) and random blood glucose $\geq 200 \mathrm{mg} / \mathrm{dl}$ or 2 hours post prandial blood glucose $\geq 200 \mathrm{mg} / \mathrm{dL} .{ }^{9}$ Chronic kidney disease was defined as estimated Glomerular Filtration Rate (eGFR) below 60 $\mathrm{ml} / \mathrm{min} / 1.73 \mathrm{~m}^{2}$ where eGFR $=((140$-age $) \times$ weights $(\mathrm{kg})) /$ $\left(72 \times\right.$ serum creatinine) ${ }^{10}$ History of myocardial infarction of each patients is recorded. Patients without the necessary data were not included in the data analysis of corresponding comorbidities.

\section{Results}

There were 128 patients who attended the Rheumatology clinic during period January 2012 - December 2013 and diagnosed with gout or ICD 10 code M10. Six patients were excluded from the data analysis due to the lack of data of interest. From these patients, $100(81.96 \%)$ patients were male and $22(18.03 \%)$ patients were female. Mean age of patients with gout was 58 with standard deviation 11.25 (Table 1).

Table 1 Patients Characteristic

\begin{tabular}{|c|c|}
\hline Variable & n (\%) \\
\hline \multicolumn{2}{|l|}{ Gender } \\
\hline Male & $100(81.96 \%)$ \\
\hline Female & 22 (18.03\%) \\
\hline Age & $58($ SD 11.25)* \\
\hline
\end{tabular}

* Mean (SD)

There were 5 patients whose data were incomplete for blood pressure. Out of 117 patients, 50 patients $(42.73 \%)$ that composed of 40 male and 10 female got hypertension. For BMI purpose, there were 32 patients who didn't have their height or weight measured. From 90 patients, 11 patients $(12.2 \%)$ who consisted of 7 male and 4 female were obese. Fifty nine patients got no data about either blood glucose measurement or Diabetes history thus weren't included in the analysis of Diabetes. Among 40 patients which included, 16 patients (25.39\%) consisted of 13 male and 3 female had Diabetes. There were 42 patients whose data were incomplete for determining eGFR, thus only 81 patients were included in the data analysis. From 43 patients (53.08\%), there were 30 men and 6 women suffered from CKD. Coronary Artery Disease (CAD) was found in 13 male and 6 female (15.70\%) out of 121 patients. (Figure 1)

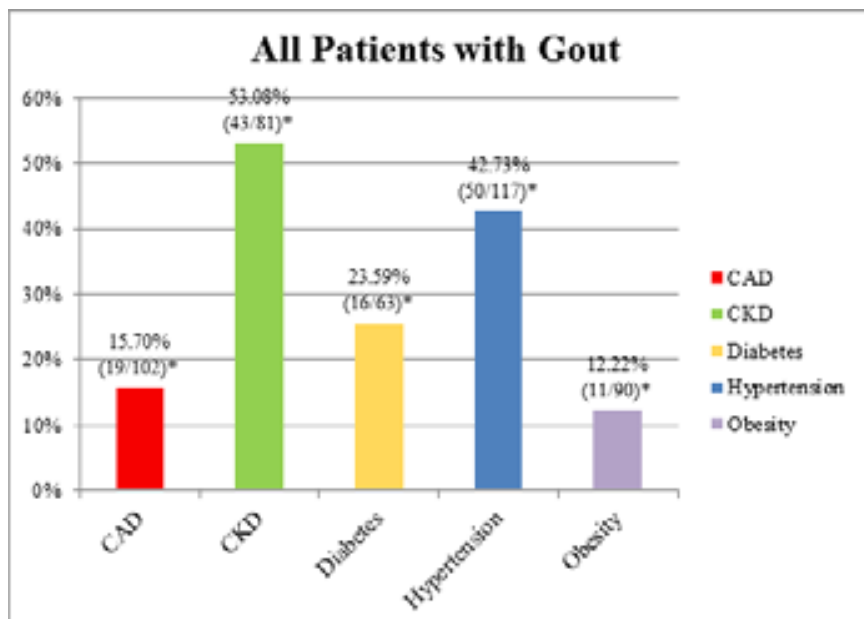

* Numbers displayed (patients with comorbidities/ all patients with particular data regarding the comorbidities)

Figure 1 Comorbidities in Patients with Gout

\section{Discussions}

In this study, patients with gout had mean age of 58 (11.25) years. Nearly similar data also were presented by Mikuls et $a l$. in their study of gout epidemiology that the mean age of patients with gout is 60.5 (15.4) years. Male predominance in this study, as commonly known in gout patients, was stated in that study as well $(79.6 \%) .{ }^{11}$

The most prevalent comorbidity in this study was CKD (53.08\%) characterized by eGFR $<60 \mathrm{ml} / \mathrm{min} / 1.73^{2}$. Different results were presented in studies carried out in United States by Zhu et al. in which hypertension (74\%) was the leading comorbidities in gouty patients in the United States (US) while CKD $(71 \%)$ was the second most. ${ }^{5}$ Many incomplete data in the analysis of CKD frequency could be the factor that influencing this discrepancies. Nevertheless, CKD had strong relation with gout based on the knowledge up to now. Kidneys act as the main excretory organs of these metabolism end products in such way that disturbance in the kidney function will decrease uric acid clearance and thus increase serum uric acid level. ${ }^{1}$ Besides that, hyperuricemia could cause a downfall in kidneys' function through urate nephropathy, uric acid nephropathy, or nephrolithiasis which terminally lead to renal failure.

Hypertension occurred in $42.73 \%$ of patients with gout in this study compared with $74 \%, 17.5 \%$, and $18.5 \%$ which stated in studies of gout comorbidities in US, United Kingdom (UK), and Germany respectively. ${ }^{5,6}$ These differences occurred probably due to the lack of data regarding anti-hypertension medication used which probably decrease the number of hypertensive patients in this study. Lifestyle variation in the population studied also contributed to these variations. Nevertheless, increased serum uric acid level is constantly associated with hypertension and many data and evidence support this. Pathogenesis of hypertension in hyperuricemia is related to the biological effects of uric acid in vascular such as to decrease Nitric Oxide (NO), increase Reactive Oxygen Species (ROS), induce vascular inflammation, and to induce proliferation of vascular smooth-muscle cells thus promote to vascular narrowing. In addition to vascular effects, uric acid 
also exerts impacts towards renal such as increased renin, decreased NO, interstitial inflammation, interstitial fibrosis which all leads to vasoreactive hypertension. ${ }^{4,12}$

Diabetes which occurred in $25.39 \%$ of all gout patients in this study is concordant with other study in Germany and US which was $25.9 \%$ and $26 \%$ respectively. ${ }^{5,6}$ Many studies had linked hyperuricemia with Diabetes Mellitus. One metaanalysis study had associated serum uric acid level with the risk of developing type 2 Diabetes and found that each $1 \mathrm{mg} /$ dl increase in serum uric acid resulted in $17 \%$ higher risk of having type 2 Diabetes Mellitus. ${ }^{13}$ Uric acid had been found as mediator of proinflammatory imbalance in adipose cells by inducing ROS production via stimulation of NADPH oxidase and decreasing adiponectin production which is an important insulin sensitizer and anti-inflammatory substance. ${ }^{14,15}$

Obesity was found in $12.22 \%$ patients with gout in this study which was smaller in frequency compared with the study in US (53\%) and UK $(27.7 \%))^{5,6}$ This discrepancy probably contributed by the lifestyle and socioeconomic level differences between the population studied. However, obesity had been associated with gout in study by Chen et al. who stated that obesity in women and hypertriglyceridemia in men could precipitate gout attacks in the absence of saturated serum uric acid level. ${ }^{16}$ It is also proved from the study in the United Kingdom general population that obesity was associated with higher risk of developing gout. ${ }^{17}$

The presence of CAD in $15.7 \%$ patients with gout in this study also stated by the studies in US, UK, and Germany $\left(11.6 \%, 7.4 \%\right.$, and $5.8 \%$ respectively). ${ }^{5,6}$ Relationship between gout and coronary artery disease could be explained by the effects of uric acid towards vascular which is to cause endothelial dysfunction through reduction of $\mathrm{NO}$ and production of ROS. ${ }^{12}$

The results of this study are consistent with previous study carried out in US, UK, and Germany about comorbidities in patients with gout such as CKD, hypertension, diabetes mellitus, $\mathrm{CAD}$, and obesity even though there were slight differences in the results obtained. Data regarding comorbidities of gout in Asia are lacking. These discrepancies probably caused by the differences in lifestyle and socioeconomic status of the population studied. Besides that, the incompleteness of data could be one of the factors affecting the differences. This study only address the most common comorbidities occurred in gout patients, while for the cause and effect relationship between gout and its comorbidities or vice versa cannot be determined. The results of this study can be used in the future as a step for further research about association between gout and its comorbidities. The consistent presence of comorbidities in patients with gout found in this study necessitates more meticulous comprehensive approach to gout management through early detection of these reported comorbidities and furthermore considering those comorbidities treatments' effects towards serum uric acid level and vice versa.

\section{REFERENCES}

1. Keenan RT, Nowatzky J, Pillinger MH. Etiology and Pathogenesis of Hyperuricemia and Gout. In: Firestein GS, Budd RC, Gabriel SE, Mcinnes IB, O'Dell JR, editors. Kelley's Textbook of Rheumatology. 9th ed. Philadelphia: Elsevier Saunders; 2013. p. 1533-53.

2. Burns CM, Wortmann RL. Clinical Features and Treatment of Gout. In: Firestein GS, Budd RC, Gabriel SE, Mcinnes IB, O’Dell JR, editors. Kelley's Textbook of Rheumatology. 9thed. Philadelphia: Elsevier Saunders; 2013. p. $1554-75$.

3. Zhu Y, Pandya BJ, Choi HK. Prevalence of gout and hyperuricemia in the US general population: the National Health and Nutrition Examination Survey 2007-2008. Arthritis Rheum 2011;63(10):3136-41.

4. Pillinger MH, Goldfarb DS, Keenan RT. Gout and its comorbidities. Bull NYU Hosp Jt Dis 2010;68(3):199-203.

5. Zhu Y, Pandya BJ, Choi HK. Comorbidities of gout and hyperuricemia in the US General Population: NHANES 2007-2008. Am J Med 2012;125(7):679-87.

6. Annemans L, Spaepen E, Gaskin M, Bonnemaire M, Malier V, Gilbert $T$, et al. Gout in the UK and Germany: prevalence, comorbidities and management in general practice 2000-2005. Ann Rheum Dis 2008;67(7):960-6.

7. Kotchen TA. Hypertensive Vascular Disease. In: Longo DL, Kasper DL, Jameson JL, Fauci AS, Hauser SL, Loscalzo J, editors. Harrison's Principle of Internal Medicine. 18 ed. United States: McGrawHill; 2012. p. 2042-59.

8. Bistrian BR, Driscoll DF. Enteral and Parenteral Nutrition Therapy. In: Longo DL, Kasper DL, Jameson JL, Fauci AS, Hauser SL, Loscalzo J, editors. Harrison's Principle of Internal Medicine. 18 ed. United States: McGrawHill; 2012. p. 612-21.

9. Powers AC. Diabetes Mellitus. In: Longo DL, Kasper DL, Jameson JL, Fauci AS, Hauser SL, Loscalzo J, editors. Harrison's Principle of Internal Medicine. 18 ed. United States: McGrawHill; 2012. p. 2968-3002.

10. Bargman JM, Skorecki K. Chronic Kidney Disease. In: Longo DL, Kasper DL, Jameson JL, Fauci AS, Hauser SL, Loscalzo J, editors. Harrison's Principle of Internal Medicine. 18 ed. United States: McGrawHill; 2012. p. 2308-21.

11. Mikuls T, Farrar J, Bilker W, Fernandes S, Schumacher H, Saag K. Gout epidemiology: results from the UK general practice research database, 1990 - 1999. Ann Rheum Dis 2005;64(2):267-72.

12. Feig DI, Kang D-H, Johnson RJ. Uric acid and cardiovascular risk. N Engl J Med 2008;359(17):1811-21.

13. Kodama S, Saito K, Yachi Y, Asumi M, Sugawara A, Totsuka K, et al. Association between serum uric acid and development of type 2 diabetes. Diabetes care 2009;32(9):1737-42.

14. Sautin YY, Nakagawa T, Zharikov S, Johnson RJ. Adverse effects of the classic antioxidant uric acid in adipocytes: NADPH oxidase-mediated oxidative/nitrosative stress. Am J Physiol Cell Physiol. 2007;293(2):C584C96.

15. Baldwin W, McRae S, Marek G, Wymer D, Pannu V, Baylis C, et al. Hyperuricemia as a mediator of the proinflammatory endocrine imbalance in the adipose tissue in a murine model of the metabolic syndrome. Diabetes 2011;60(4):1258-69.

16. Chen JH, Pan WH, Hsu CC, Yeh WT, Chuang SY, Chen PY, et al. Impact of obesity and hypertriglyceridemia on gout development with or without hyperuricemia: a prospective study. Arthritis Care Res 2013;65(1):13340.

17. Soriano LC, Rothenbacher D, Choi HK, Rodríguez LAG. Contemporary epidemiology of gout in the UK general population. Arthritis Res Ther 2011;13(2):R39. 